\title{
Role of Cationic Size and Valency in Mechanoelectrical Transduction of Ion Containing Polymers
}

Hamad A. Albehaijan ${ }^{1,2}$, Jinwei Cao ${ }^{1}$, Camilo R. Piedrahita ${ }^{1}$, Antal Jákli ${ }^{3}$, and Thein Kyu ${ }^{1 *}$ ${ }^{1}$ Department of Polymer Engineering, University of Akron, Akron, OH 44325, USA

${ }^{2}$ SABIC Plastic Applications Development Center, Riyadh 12373, Kingdom of Saudi Arabia

${ }^{3}$ Department of Physics, Advanced Materials and Liquid Crystal Institute, Kent State University, Kent $\mathrm{OH} 44242$, USA

Corresponding author email: tkyu@uakron.edu

- 7 Pages

- 4 Figures 


\section{Supplementary Information}

As shown in Figure S1a, formation of $\mathrm{Al}(\mathrm{TFSI})_{3}$ can be confirmed via FTIR where infrared spectrum of HTFSI acid precursors and $\mathrm{Al}(\mathrm{TFSI})_{3}$ powder was obtained to study the salt formation by monitoring the characteristic IR peaks corresponding to the $\mathrm{N}-\mathrm{H}$ bonds and the behavior of different functionalities of TFSI- over the range of $400-1500 \mathrm{~cm}^{-1}$. It was evident that the IR peak corresponding to the $\mathrm{N}-\mathrm{H}$ bond at $1440 \mathrm{~cm}^{-1}$ was fully consumed upon metal/acid reaction HTFSI with aluminum metal suggestive of complete ionization of HTFSI resulting in dissociated TFSIanions which subsequently neutralized by aluminum to produce $\mathrm{Al}(\mathrm{TFSI})_{3}$ salt. Other peaks, in the range of $400 \mathrm{~cm}^{-1}$ to $1500 \mathrm{~cm}^{-1}$, corresponding to TFSI- anion functionalities were shifted to different energy levels and higher intensity upon aluminum neutralization, such as C-F (at 497 $\left.\mathrm{cm}^{-1}\right)$ and $\mathrm{SO}_{2}\left(563 \mathrm{~cm}^{-1}\right)$ functions, driven by a higher population of TFSI- anions in $\mathrm{Al}(\mathrm{TFSI})_{3}$ as compared to HTFSI and the imposed constrains provided upon Al neutralization. Figure S1b shows additional confirmation of $\mathrm{Al}(\mathrm{TFSI})_{3}$ salt formation via metal/acid neutralization, where EDX profile of the resultant powder was collected to confirm the presence of the building elements $\mathrm{Al}(\mathrm{TFSI})_{3}$. EDX reveals the presence of $\mathrm{Al}$ cation as indicated by the energy peak located at 1.48 $\mathrm{keV}$, as elements of TFSI- anion were indicated in their respective energy levels.
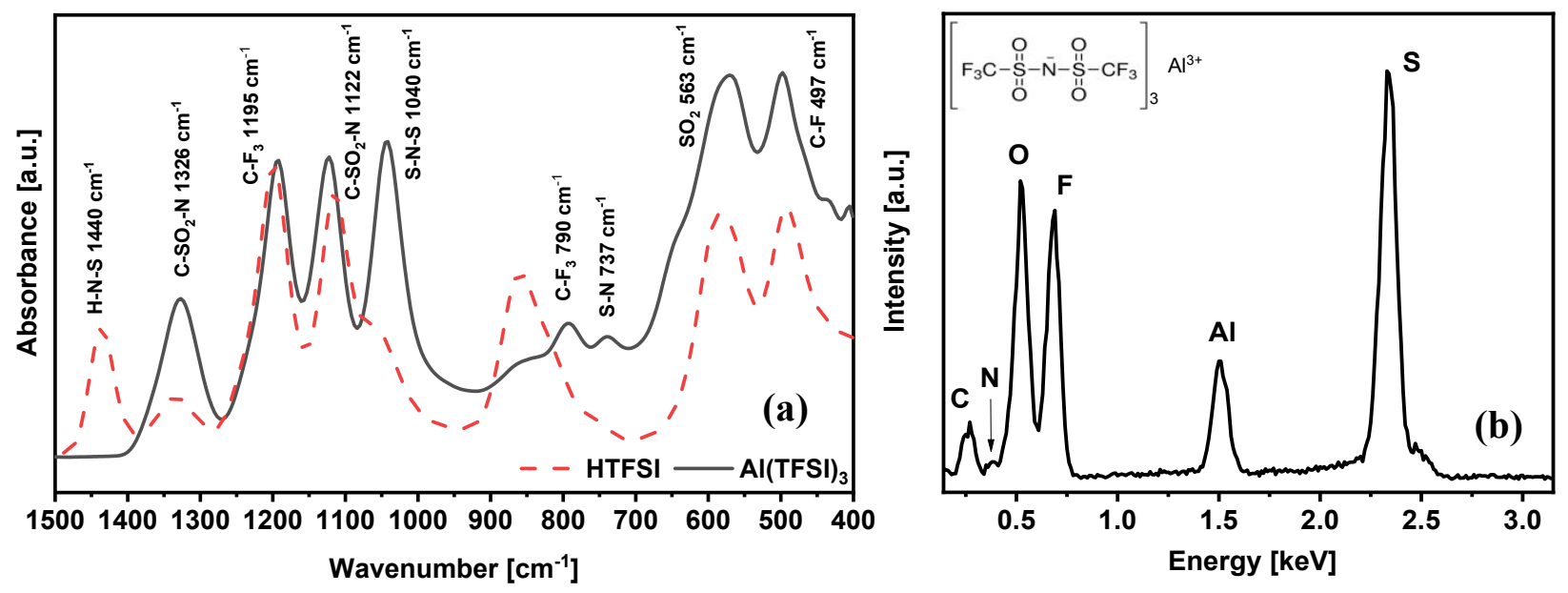
Figure S1. (a) FTIR analysis of neat HTFSI acid and as-synthesized $\mathrm{Al}(\mathrm{TFSI})_{3}$ salt by metal/acid reaction, and (b) EDX profile of synthesized Al(TFSI)3. The energy shift of TFSI- functionality and presence of $\mathrm{Al}^{+3}$ indicate the formation of $\mathrm{Al}(\mathrm{TFSI})_{3}$.

Compositions of LiTFSI containing PEMs were made to determine the optimum ion conducting membrane as a control sample by varying salt content from $5-30 \mathrm{wt} \%$. As seen in Figure S2, PEMs of $10 \%$ salt content demonstrate the highest conductivity level, similar findings were demonstrated by Ruixuan et al ${ }^{1}$.

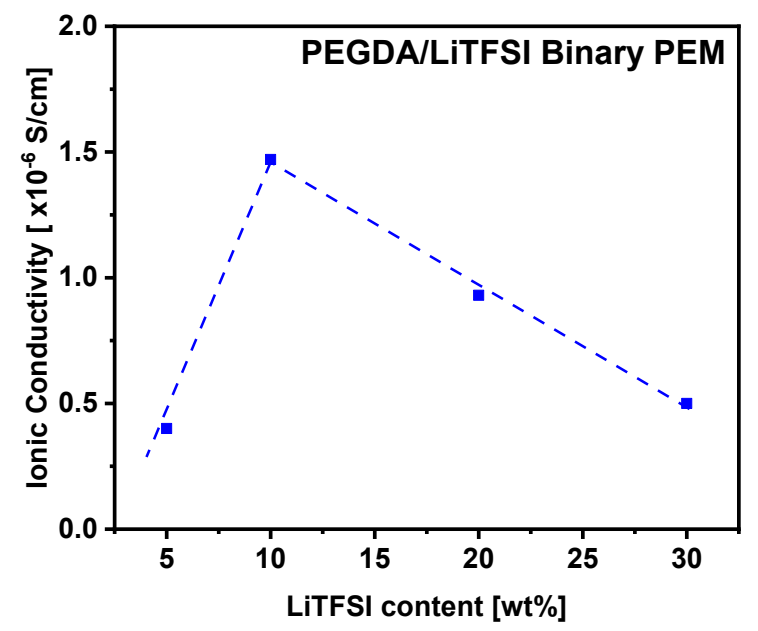

Figure S2. Ionic conductivity of PEGDA/LiTFSI binary PEMs as a function of lithium salt content of 5-30 wt $\%$.

Infrared spectrums were acquired for neat-PEGDA network and salt containing PEMs to study the effect of physical coordination (i.e. ion-dipole interactions) of solvated cations on and ether constituents on the final configuration of PEGDA covalent network. Figure S3 shows a shift of the characteristic peak at $1049 \mathrm{~cm}^{-1}$ corresponding to C-O-C asymmetric stretching to higher energy, and broadening of C-H symmetric stretching peak located at $2870 \mathrm{~cm}^{-1}$ of PEGDA network, upon salt addition, which may be attributed to the higher restriction resultant from cation-dipole 
interactions especially in the case of PEGDA/Al(TFSI) $)_{3}$ since $\mathrm{Al}^{+3}$ has the largest charge/size ratio compared to other cations in the present study. Behavior of peaks corresponding to salt anions, i.e. TFSI-, were monitored in this study as well, where $\mathrm{SO}_{2}$ symmetric vibration located at $1350 \mathrm{~cm}^{-1}$ and $\mathrm{CF}_{3}$ located at $1188 \mathrm{~cm}^{-1}$ remained unchanged upon the addition of different salts since their molar amount was the same in all cases. Similar observations of LiTFSI salt addition to PEO and PEGDA networks were previously reported ${ }^{2-4,4}$.

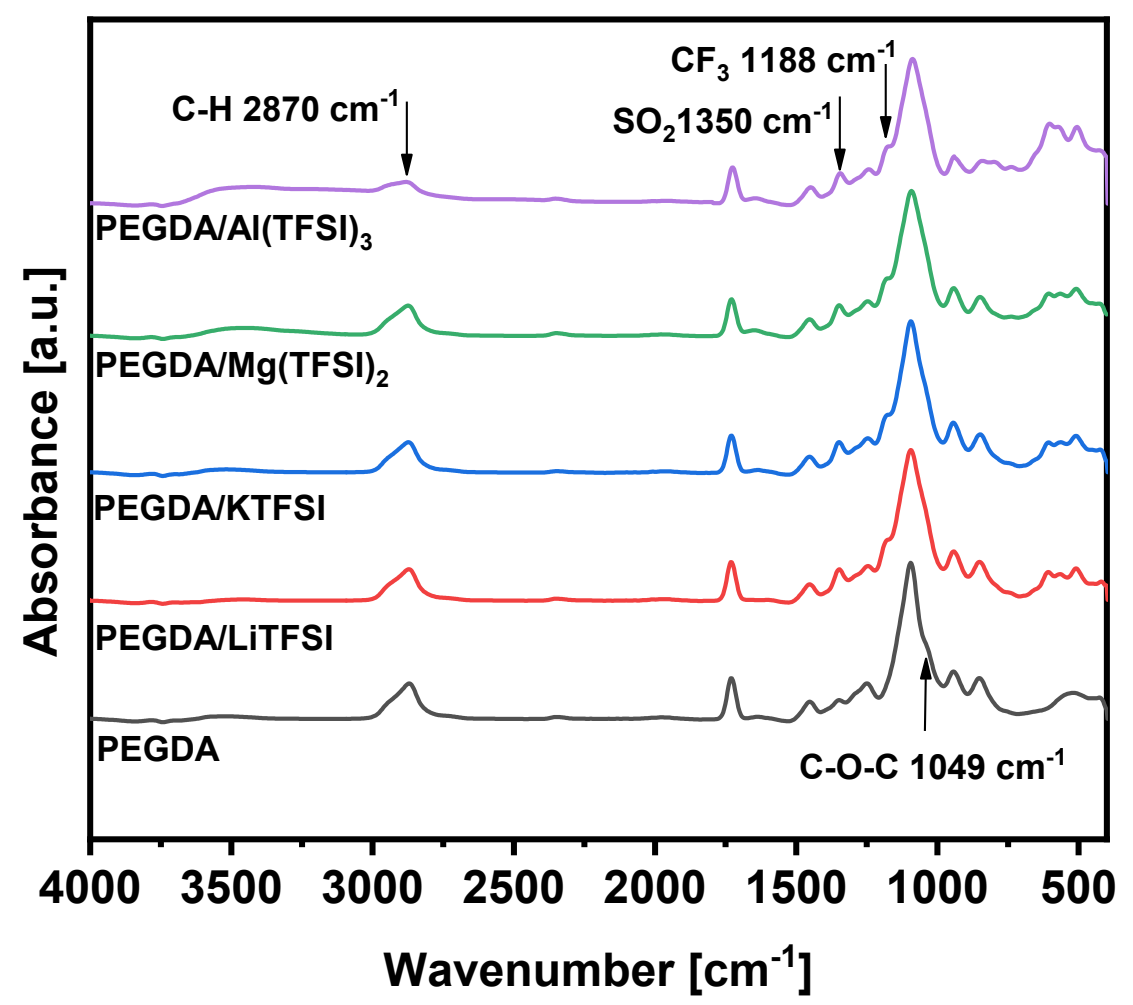

Figure S3. Infrared spectra of neat UV-crosslinked PEGDA network and PEMs based on PEGDA/salt 90/10 mixtures of LiTFSI, KTFSI, $\mathrm{Mg}(\mathrm{TFSI})_{2}$ and $\mathrm{Al}(\mathrm{TFSI})_{3}$, showing the movement of IR bands corresponding to $\mathrm{C}-\mathrm{O}-\mathrm{C}, \mathrm{SO}_{2}$ and $\mathrm{CF}_{3}$ with increasing cationic size and valency and broadening C-H band suggestive of polyether / metal ion coordination. 
Furthermore, the PEMs' glass transition temperatures, $T_{g}$, was investigated to determine the effect of cation size and valency on the elastic properties of the present flexible PEMs, in relation to their flexoelectric characteristics. Figure S4a depicts the PEMs' dual network dependence of $T_{g}$ as a function of salt as determined by DSC. Distinctive deflections of thermograms of PEMs were observed in neat PEGDA-network [indicated by PEGDA] and salts containing PEMs. These deflections reflect the $T_{g}$ of PEMs, which indicate the transition of the present network from the glassy to rubbery state. The $T_{g}$ of neat-PEGDA covalent network was found to be located at $-41.6{ }^{\circ} \mathrm{C}$, where an increase of $\mathrm{T}_{\mathrm{g}}$ was observed upon the introduction the secondary physical network, viz. ion-dipole coordination network, upon salt addition. The increase in $T_{g}$ indicates larger restrictions on the polymer chain mobility, which might be imposed by the added physical network, and thus observed higher $\mathrm{T}_{\mathrm{g}}$ values. It was found that PEMs containing larger cations, exhibited a higher $T_{g}$ as observed in $\mathrm{Li}^{+}$vs. $\mathrm{K}^{+}$. Similarly, a gradual increase of $\mathrm{T}_{\mathrm{g}}$ was observed upon increasing the cation valency from monovalent to trivalent, e.g., $\mathrm{Li}^{+}$vs. $\mathrm{Mg}^{2+}$ vs. $\mathrm{Al}^{3+}$. Increasing cation size and valency resulted in higher levels of steric hindrance driven by ion-dipole coordination, thereby imposing constraints on the PEGDA covalent network and promoting tighter network as manifested by a higher $\mathrm{T}_{\mathrm{g}}$. DMA was also conducted to elucidate the influence of cation size and valency on $\mathrm{T}_{\mathrm{g}}$ of present PEMs. Shown in Figure S4b is the dependency of loss tangent, $\tan \delta$, as a function of temperature of the aforementioned PEMs. Loss $\tan \delta$ peaks were observed for neat-PEGDA network as well as salt containing PEMs at the characteristic temperatures representing their respective $T_{g}$. As evidenced by DMA, $T_{g}$ followed similar trends as observed in DSC (Figure S4a), where addition of salt resulted in chain mobility reduction due to greater steric hindrance and larger levels of ion-dipole coordination. For instant, neat-PEGDA network showed a $\mathrm{T}_{\mathrm{g}}$ (i.e. $\tan \delta$ peak) of $-23.7^{\circ} \mathrm{C}$, followed by an increase of $\mathrm{T}_{\mathrm{g}}$ 
upon salt addition with the highest value obtained at $-7.2{ }^{0} \mathrm{C}$ for $\mathrm{Al}^{3+}$ containing PEM [labelled by PEGDA/Al(TFSI) ${ }_{3}$ ] since it has the largest valency in the present PEM systems, thus the highest degree of coordination. These observations implied that cations of larger size and/or valency limit their use in lower temperature environments, for applications that require flexibility, where bending is the driving stimuli of mechanoelectrical transduction in wearable devices, wind and oceanic wave motions.
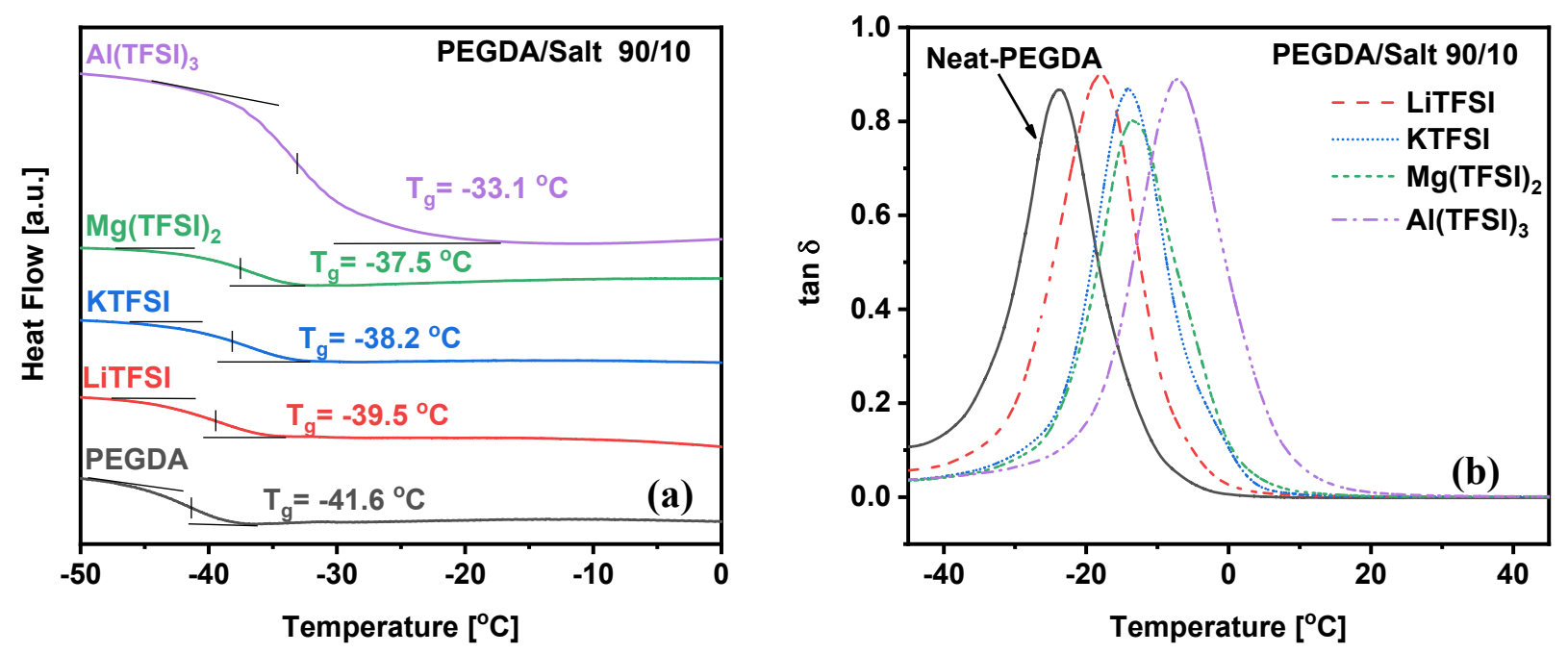

Figure S4. (a) Glass transition temperature $\left(\mathrm{T}_{\mathrm{g}}\right)$ variation of neat-PEGDA covalent network and binary PEMs based on PEGDA/Salt 90/10 composition using various salts of different cation sizes and valencies, and (b) the loss tangent variations as a function of temperature, where distinctive peaks indicate $T_{g}$ of PEMs. The $T_{g}$ was found to increase with the addition of metal salts exhibiting the systematic movement of $\mathrm{T}_{\mathrm{g}}$ with the cation size and valency ratios.

\section{References}

(1) He, R.; Kyu, T. Effect of Plasticization on Ionic Conductivity Enhancement in Relation to Glass Transition Temperature of Crosslinked Polymer Electrolyte Membranes.

Macromolecules 2016, 49 (15), 5637-5648. 
(2) Rey, I.; Lassegues, J. C.; Grondin, J.; Servant, L. Infrared and Raman Study of the PEOLiTFSI Polymer Electrolyte. Electrochim. Acta 1998, 43 (10-11), 1505-1510.

(3) Echeverri, M.; Hamad, C.; Kyu, T. Highly Conductive , Completely Amorphous Polymer Electrolyte Membranes Fabricated through Photo-Polymerization of Poly ( Ethylene Glycol Diacrylate ) in Mixtures of Solid Plasticizer and Lithium Salt. Solid State Ionics 2014, 254, 92-100.

(4) He, R.; Echeverri, M.; Ward, D.; Zhu, Y.; Kyu, T. Highly Conductive Solvent-Free Polymer Electrolyte Membrane for Lithium-Ion Batteries : Effect of Prepolymer Molecular Weight. J. Memb. Sci. 2016, 498, 208-217. 